\title{
A Geometric Integration Based on Magnus Series Expansion for Human T-Cell Lymphotropic Virus I (HTLV-I) Infection of $\mathrm{CD}^{+}$T-Cells Model
}

\author{
M. Tarık Atay ${ }^{1}$, Musa Başbük $^{2}$ and Aytekin Eryılmaz ${ }^{2 *}$ \\ ${ }^{1}$ Department of Mechanical Engineering, Abdullah Gül University, 38080, Kayseri/Turkey \\ ${ }^{2}$ Department of Mathematics, Nevşehir Haci Bektaş Veli University, 50300, Nevşehir/Turkey \\ Email: eryilmazaytekin@gmail.com
}

\begin{abstract}
In this paper, we investigated a numeric integration based on Magnus series expansion namely Magnus Series Expansion Method (NMG) for nonlinear Human T-Cell Lymphotropic Virus I (HTLV-I) infection of CD4 ${ }^{+}$T-cells model. Fourth order Magnus series expansion method (NMG4) and explicit Runge-Kutta (RK45) are used to obtain numerical solutions of HTLV-I infection of CD4 ${ }^{+}$T-cells model. The results obtained by NMG4 and RK45 are compared.
\end{abstract}

Keywords: Non-linear Differential Equation System, Magnus Series Expansion Method, Geometric Integration, Lie Group Method.

\section{Introduction}

Dynamics of Human T-Cell Lymphotropic Virus I (HTLV-I) infection of CD4 ${ }^{+}$T-cells model examined in $[1-7]$ is,

$$
\begin{aligned}
\frac{d T}{d t} & =\lambda-\mu_{T} T-k T_{A} T \\
\frac{d T_{L}}{d t} & =k T_{A} T-\left(\mu_{L}+\alpha\right) T_{L} \\
\frac{d T_{A}}{d t} & =\alpha T_{L}-\left(\mu_{A}+\rho\right) T_{A} \\
\frac{d T_{M}}{d t} & =\rho T_{A}+\beta T_{M}\left(1-T_{M} / T_{\max }\right)-\mu_{M} T_{M}
\end{aligned}
$$

with the initial conditions,

$$
T(0)=P_{1}, \quad T_{L}(0)=P_{2}, \quad T_{A}(0)=P_{3}, \quad T_{M}(0)=P_{4},
$$

where $T(t), T_{L}(t), T_{A}(t), T_{M}(t)$ denote the concentration of healthy CD4 ${ }^{+}$T-cells at time $t$, the concentration of latently infected $\mathrm{CD} 4^{+} \mathrm{T}$-cells, the concentration of actively infected CD4 ${ }^{+}$T-cells and the concentration of leukemic cells at time $t$ respectively. The parameters $\lambda, \mu_{T}$ and $k$ denote the natural date rate of $\mathrm{CD} 4^{+} \mathrm{T}$-cells, the rate at which uninfected cells are contacted by actively infected cells, the rate of infection of T-cells with virus from actively infected cells respectively. $\mu_{L}, \mu_{A}, \mu_{M}$ are blanket death terms for latently infected, actively infected and leukemic cells respectively. $\alpha$ and $\rho$ denote the rates at which latently infected and actively infected cells become actively infected and leukemic respectively. The rate $\beta$ determines the the speed at which the saturation level for leukemia cells is reached. $T_{\max }$ is the maximal value that adult T-cell leukemia can reach [7].

The purpose of this paper is to obtain numerical solution of the system (1-4) subject to the initial conditions (5) by using a structure preserving numerical integrator based on Magnus Expansion, namely Magnus Expansion Method.

In 1954, Magnus [8] provided an exponential representation of the solution of a first order linear homogeneous differential equation for a linear operator that was named Magnus Expansion after him. 
Since 1960's the Magnus expansion has been successfully applied in various areas of physics and chemistry (see [9] for a list of references). Iserles and Norsett (1997) [10,11] presented a practical recursive algorithm that generated the terms of Magnus expansion. Blanes et al. (1998) [12] considered the approximate solutions of matrix linear differential equations by matrix exponentials and the convergence issue of Magnus and Fer expansions. They obtained the upper bounds for the convergence radius in terms of the norm of the defining matrix of the system. Moan and Niesen (2008) [13] considered the question: When does the series converge? The main result they obtained, established a necessary condition for convergence.

\section{Magnus Expansion Method}

The linear differential equation on a matrix Lie-group is an equation of the form

$$
Y^{\prime}=A(t) Y, \quad t \geq 0, \quad Y(0)=Y_{0} \in \mathcal{G},
$$

where $A: \mathcal{G} \longrightarrow \mathcal{R}$ is the matrix function, $\mathcal{G}$ is the Lie group, $g$ is the Lie algebra of the corresponding Lie-group $\mathcal{G}$. Magnus expressed the solution of equation (6) as the exponential of a certain function [8],

$$
Y(t)=e^{\Omega(t)}
$$

and obtained an infinite recursive series for $\Omega$ as follows,

$$
\begin{gathered}
\Omega_{0} \equiv 0 \\
\Omega_{n+1}=\int_{0}^{t} d \exp _{\Omega_{n}}^{-1} A(\xi) d \xi=\sum_{k=0}^{\infty} \frac{B_{k}}{k !} \int_{0}^{t} a d_{\Omega_{n}}^{k} A(\xi) d \xi, \quad n=0,1,3, \ldots
\end{gathered}
$$

where $B_{k}$ are the Bernoulli numbers. Substituting the equation (8) into the equation (9) one can get the $\Omega_{i}$ for $i=0,1,2,3, \ldots$, respectively

$$
\begin{aligned}
\Omega_{1}= & \int_{0}^{t} A\left(t_{1}\right) d t_{1} \\
\Omega_{2}= & \int_{0}^{t} A\left(t_{1}\right) d t_{1}-\frac{1}{2} \int_{0}^{t}\left[\int_{0}^{t_{2}} A\left(t_{1}\right) d t_{1}, A\left(t_{2}\right)\right] d t_{2}+\ldots \\
\Omega_{3}= & \int_{0}^{t} A\left(t_{1}\right) d t_{1}-\frac{1}{2} \int_{0}^{t}\left[\int_{0}^{t_{2}} A\left(t_{1}\right) d t_{1}, A\left(t_{2}\right)\right] d t_{2} \\
& +\frac{1}{12} \int_{0}^{t}\left[\int_{0}^{t_{3}} A\left(t_{1}\right) d t_{1},\left[\int_{0}^{t_{3}} A\left(t_{1}\right) d t_{1}, A\left(t_{3}\right)\right]\right] d t_{3} \\
& +\frac{1}{4} \int_{0}^{t}\left[\int_{0}^{t_{3}}\left[\int_{0}^{t_{2}} A\left(t_{1}\right) d t_{1}, A\left(t_{2}\right)\right] d t_{2}, A\left(t_{3}\right)\right] d t_{3}+\ldots
\end{aligned}
$$

The Magnus series expansion is,

$$
\Omega(t)=\sum_{k=0}^{\infty} H_{k}(t),
$$

where each $H_{k}$ includes exactly $k+1$ integrals and $k$ commutators [14].

Thus,

$$
\begin{aligned}
& H_{1}=\int_{0}^{t} A\left(t_{1}\right) d t_{1} \\
& H_{2}=-\frac{1}{2} \int_{0}^{t}\left[\int_{0}^{t_{2}} A\left(t_{1}\right) d t_{1}, A\left(t_{2}\right)\right] d t_{2}
\end{aligned}
$$




$$
\begin{aligned}
H_{3}= & \frac{1}{12} \int_{0}^{t}\left[\int_{0}^{t_{3}} A\left(t_{1}\right) d t_{1},\left[\int_{0}^{t_{3}} A\left(t_{1}\right) d t_{1}, A\left(t_{3}\right)\right]\right] d t_{3} \\
& +\frac{1}{4} \int_{0}^{t}\left[\int_{0}^{t_{3}}\left[\int_{0}^{t_{2}} A\left(t_{1}\right) d t_{1}, A\left(t_{2}\right)\right] d t_{2}, A\left(t_{3}\right)\right] d t_{3} \\
H_{4}= & -\frac{1}{24} \int_{0}^{t}\left[\int_{0}^{t_{4}} A\left(t_{1}\right) d t_{1},\left[\int_{0}^{t_{4}}\left[\int_{0}^{t_{2}} A\left(t_{1}\right) d t_{1}, A\left(t_{2}\right)\right] d t_{2}, A\left(t_{4}\right)\right]\right] d t_{4} \\
& -\frac{1}{24} \int_{0}^{t}\left[\int_{0}^{t_{4}}\left[\int_{0}^{t_{2}} A\left(t_{1}\right) d t_{1}, A\left(t_{2}\right)\right] d t_{2},\left[\int_{0}^{t_{4}} A\left(t_{1}\right) d t_{1}, A\left(t_{4}\right)\right]\right] d t_{4} \\
& -\frac{1}{24} \int_{0}^{t}\left[\int_{0}^{t_{4}}\left[\int_{0}^{t_{3}} A\left(t_{1}\right) d t_{1},\left[\int_{0}^{t_{3}} A\left(t_{1}\right) d t_{1}, A\left(t_{3}\right)\right]\right] d t_{3}, A\left(t_{4}\right)\right] d t_{4} \\
& -\frac{1}{8} \int_{0}^{t}\left[\int_{0}^{t_{4}}\left[\int_{0}^{t_{3}}\left[\int_{0}^{t_{2}} A\left(t_{1}\right) d t_{1}, A\left(t_{2}\right)\right] d t_{2}, A\left(t_{3}\right)\right] d t_{3}, A\left(t_{4}\right)\right] d t_{4} .
\end{aligned}
$$

By using multivariate Gaussian quadrature, Casas \& Iserles [15] presented algorithms for third and fourth order Magnus Expansion Method (NMG3 and NMG4) for nonlinear Lie type differential equations as follows, Third order Magnus Expansion Method for nonlinear equations (NMG3)

$$
\begin{aligned}
u_{1} & =0 \\
k_{1} & =h A\left(0, Y_{0}\right) \\
u_{2} & =\frac{1}{2} k_{1} \\
k_{2} & =h A\left(\frac{h}{2}, e^{u_{2}} Y_{0}\right) \\
u_{3} & =\frac{1}{4}\left(k_{1}+k_{2}\right) \\
k_{3} & =h A\left(\frac{h}{2}, e^{u_{3}} Y_{0}\right) \\
u_{4} & =k_{2} \\
k_{4} & =h A\left(h, e^{u_{4}} Y_{0}\right) \\
v_{3} & =\frac{1}{6}\left(k_{1}+4 k_{3}+k_{4}\right)-\frac{1}{3}\left[u_{3}, k_{3}\right]-\frac{1}{12}\left[u_{4}, k_{4}\right] \\
Y_{1}(t) & =e^{v_{3}} Y_{0} .
\end{aligned}
$$

Fourth order Magnus Expansion Method for nonlinear equations (NMG4)

$$
\begin{aligned}
u_{1} & =0 \\
k_{1} & =h A\left(t_{n}, Y_{n}\right) \\
Q_{1} & =k_{1} \\
u_{2} & =\frac{1}{2} Q_{1} \\
k_{2} & =h A\left(t_{n}+\frac{h}{2}, e^{u_{2}} Y_{0}\right) \\
Q_{2} & =k_{2}-k_{1} \\
u_{3} & =\frac{1}{2} Q_{1}+\frac{1}{4} Q_{2} \\
k_{3} & =h A\left(t_{n}+\frac{h}{2}, e^{u_{3}} Y_{0}\right) \\
Q_{3} & =k_{3}-k_{2} \\
u_{4} & =Q_{1}+Q_{2}
\end{aligned}
$$




$$
\begin{aligned}
k_{4} & =h A\left(t_{n}+h, e^{u_{4}} Y_{0}\right) \\
Q_{4} & =k_{4}-2 k_{2}+k_{1} \\
u_{5} & =\frac{1}{2} Q_{1}+\frac{1}{4} Q_{2}+\frac{1}{3} Q_{3}-\frac{1}{24} Q_{4}-\frac{1}{48}\left[Q_{1}, Q_{2}\right] \\
k_{5} & =h A\left(t_{n}+\frac{h}{2}, e^{u_{5}} Y_{0}\right) \\
Q_{5} & =k_{5}-k_{2} \\
u_{6} & =Q_{1}+Q_{2}+\frac{2}{3} Q_{5}+\frac{1}{6} Q_{4}-\frac{1}{6}\left[Q_{1}, Q_{2}\right] \\
k_{6} & =h A\left(t_{n}+h, e^{u_{6}} Y_{0}\right) \\
Q_{6} & =k_{6}-2 k_{2}+k_{1} \\
v & =Q_{1}+Q_{2}+\frac{2}{3} Q_{5}+\frac{1}{6} Q_{6}-\frac{1}{6}\left[Q_{1}, Q_{2}-Q_{3}+Q_{5}+\frac{1}{2} Q_{6}\right] \\
Y_{n+1} & =e^{v} Y_{n} .
\end{aligned}
$$

\section{Numerical Results}

In this section, NMG4 and the fourth order explicit Rungeâ̆ T-Cell Lymphotropic Virus I (HTLV-I) infection of CD4 ${ }^{+}$T-cells model by Mathematica 7 . Throughout this paper we set $\mu_{T}=0.66 \mathrm{~mm}^{3} /$ day, $\mu_{L}=0.06$ day, $\mu_{A}=0.05$ day, $\mu_{M}=0.005$ day, $k=0.5, \lambda=0.6$, $\alpha=0.004$ day, $\beta=0.0003$ day, $\rho=0.00004$ day and $T_{\max }=2200 \mathrm{~mm}^{3}$.

$$
\begin{aligned}
\frac{d T}{d t} & =\lambda-\mu_{T} T-k T_{A} T \\
\frac{d T_{L}}{d t} & =k T_{A} T-\left(\mu_{L}+\alpha\right) T_{L} \\
\frac{d T_{A}}{d t} & =\alpha T_{L}-\left(\mu_{A}+\rho\right) T_{A} \\
\frac{d T_{M}}{d t} & =\rho T_{A}+\beta T_{M}\left(1-T_{M} / T_{\max }\right)-\mu_{M} T_{M}
\end{aligned}
$$

with the initial conditions,

$$
T(0)=1000, \quad T_{L}(0)=250, \quad T_{A}(0)=1.5, \quad T_{M}(0)=0,
$$

By using the following transformation [16],

$$
T=p, \quad T_{L}=q, \quad T_{A}=r, \quad T_{M}=s,
$$

the equation system (14-17) yields the Lie-type matrix equation,

$$
\left[\begin{array}{c}
p \\
q \\
r \\
s \\
1
\end{array}\right]^{\prime}=\left[\begin{array}{ccccc}
-\mu_{T}-k r & 0 & 0 & 0 & \lambda \\
k r & -\mu_{L}-\alpha & 0 & 0 & 0 \\
0 & \alpha & -\mu_{A}-\rho & 0 & 0 \\
0 & 0 & \rho & \beta\left(1-\frac{s}{T_{\max }}\right)-\mu_{A} & 0 \\
0 & 0 & 0 & 0 & 0
\end{array}\right]\left[\begin{array}{c}
p \\
q \\
r \\
s \\
1
\end{array}\right]
$$

where prime denotes derivative with respect to time $t$.

$$
\text { If we call }\left[\begin{array}{c}
p \\
q \\
r \\
s \\
1
\end{array}\right] \text { as } Y \text { and }\left[\begin{array}{ccccc}
-\mu_{T}-k r & 0 & 0 & 0 & \lambda \\
k r & -\mu_{L}-\alpha & 0 & 0 & 0 \\
0 & \alpha & -\mu_{A}-\rho & 0 & 0 \\
0 & 0 & \rho & \beta\left(1-\frac{s}{T_{\max }}\right)-\mu_{A} & 0 \\
0 & 0 & 0 & 0 & 0
\end{array}\right] \text { as } A \text {, then equation }
$$

system (14-17) takes the form,

$$
Y^{\prime}=A Y .
$$


By using the algorithm (13) and conducting the iteration for the time interval $(0,1000)$ with the step size $\frac{1}{10}$, the following results are obtained. Table 1-4. compare the NMG4 and RK45 solutions for the time interval $(0,20)$ with the step size $\frac{1}{10}$ for the equation system (14-17). The obtained results for $T(t), T_{L}(t), T_{A}(t)$ and $T_{M}(t)$ are given in Fig. 1-13.

Table 1. NMG4 and RK45 solutions of $T(t)$ for the time interval $(0,20)$ with the step size $\frac{1}{10}$ for the equation system (14-17).

\begin{tabular}{cccc}
\hline$t$ & NMG4 solution & RK45 solution & Absolute Difference \\
\hline 0 & 1000 & 1000 & 0 \\
2 & 7.13582 & 7.13582 & $4.12047 \times 10^{-6}$ \\
4 & 0.096573 & 0.096573 & $6.62869 \times 10^{-6}$ \\
6 & 0.0723808 & 0.0723808 & $6.33054 \times 10^{-7}$ \\
8 & 0.0610988 & 0.0610988 & $5.42661 \times 10^{-7}$ \\
10 & 0.0549001 & 0.0549001 & $4.25295 \times 10^{-7}$ \\
12 & 0.0513063 & 0.0513063 & $3.03046 \times 10^{-7}$ \\
14 & 0.049261 & 0.049261 & $1.8866 \times 10^{-7}$ \\
16 & 0.048239 & 0.048239 & $8.85135 \times 10^{-8}$ \\
18 & 0.047952 & 0.047952 & $4.96236 \times 10^{-9}$ \\
20 & 0.0482307 & 0.0482307 & $5.93419 \times 10^{-8}$ \\
\hline
\end{tabular}

Table 2. NMG4 and RK45 solutions of $T_{L}(t)$ for the time interval $(0,20)$ with the step size $\frac{1}{10}$ for the equation system (14-17).

\begin{tabular}{cccc}
\hline$t$ & NMG4 solution & RK45 solution & Absolute Difference \\
\hline 0 & 250 & 250 & 0 \\
2 & 796.75 & 796.75 & $7.44233 \times 10^{-5}$ \\
4 & 707.304 & 707.304 & $6.10276 \times 10^{-5}$ \\
6 & 623.37 & 623.37 & $5.38087 \times 10^{-5}$ \\
8 & 549.53 & 549.53 & $4.73637 \times 10^{-5}$ \\
10 & 484.566 & 484.566 & $4.16416 \times 10^{-5}$ \\
12 & 427.411 & 427.411 & $3.6581 \times 10^{-5}$ \\
14 & 377.125 & 377.125 & $3.21183 \times 10^{-5}$ \\
16 & 332.882 & 332.882 & $2.81897 \times 10^{-5}$ \\
18 & 293.955 & 293.955 & $2.47478 \times 10^{-5}$ \\
20 & 259.704 & 259.703 & $2.17017 \times 10^{-5}$ \\
\hline
\end{tabular}

Table 3. NMG4 and RK45 solutions of $T_{A}(t)$ for the time interval $(0,20)$ with the step size $\frac{1}{10}$ for the equation system (14-17).

\begin{tabular}{cccc}
\hline$t$ & NMG4 solution & RK45 solution & Absolute Difference \\
\hline 0 & 1.5 & 1.5 & \\
2 & 6.39744 & 6.39744 & $1.79348 \times 10^{-7}$ \\
4 & 11.5167 & 11.5167 & $6.37668 \times 10^{-7}$ \\
6 & 15.4728 & 15.4728 & $1.00891 \times 10^{-7}$ \\
8 & 18.453 & 18.453 & $1.29307 \times 10^{-6}$ \\
10 & 20.6224 & 20.6224 & $1.50456 \times 10^{-6}$ \\
12 & 22.1215 & 22.1215 & $1.65579 \times 10^{-6}$ \\
14 & 23.0698 & 23.0698 & $1.75723 \times 10^{-6}$ \\
16 & 23.5689 & 23.5689 & $1.81803 \times 10^{-6}$ \\
18 & 23.7046 & 23.7046 & $1.84408 \times 10^{-6}$ \\
20 & 23.5496 & 23.5496 & $1.84767 \times 10^{-6}$ \\
\hline
\end{tabular}


Table 4. NMG4 and RK45 solutions of $T_{M}(t)$ for the time interval $(0,20)$ with the step size $\frac{1}{10}$ for the equation system (14-17).

\begin{tabular}{cccc}
\hline$t$ & NMG4 solution & RK45 solution & Absolute Difference \\
\hline 0 & 0 & 0 & 0 \\
2 & 0.000290763 & 0.000290763 & $5.77703 \times 10^{-11}$ \\
4 & 0.00100986 & 0.00100986 & $2.48410 \times 10^{-11}$ \\
6 & 0.00208226 & 0.00208226 & $4.16242 \times 10^{-11}$ \\
8 & 0.00341953 & 0.00341953 & $1.33443 \times 10^{-10}$ \\
10 & 0.00494826 & 0.00494826 & $2.44045 \times 10^{-10}$ \\
12 & 0.00660783 & 0.00660783 & $3.67955 \times 10^{-10}$ \\
14 & 0.00834854 & 0.00834854 & $5.00714 \times 10^{-10}$ \\
16 & 0.01013 & 0.01013 & $6.38569 \times 10^{-10}$ \\
18 & 0.0119194 & 0.0119194 & $7.79346 \times 10^{-10}$ \\
20 & 0.0136909 & 0.0136909 & $9.17819 \times 10^{-10}$ \\
\hline
\end{tabular}
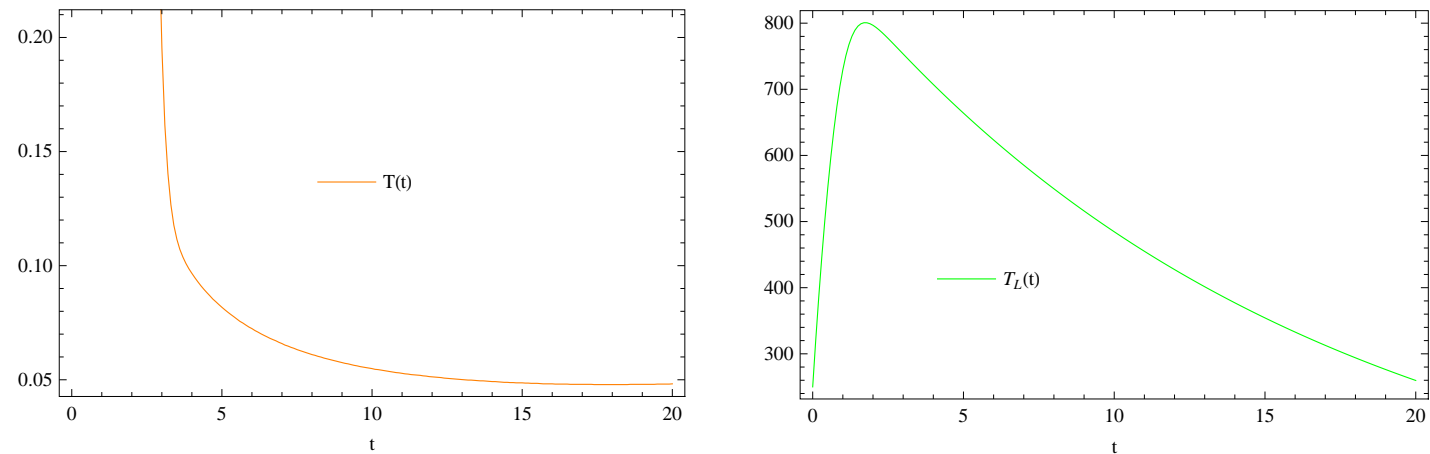

Figure 1. NMG4 solutions of $T(t)$ for the time in- Figure 2. NMG4 solutions of $T_{L}(t)$ for the time interval $(0,20)$ with the step size $\frac{1}{10}$ for the equation terval $(0,20)$ with the step size $\frac{1}{10}$ for the equation system (14-17) system (14-17)
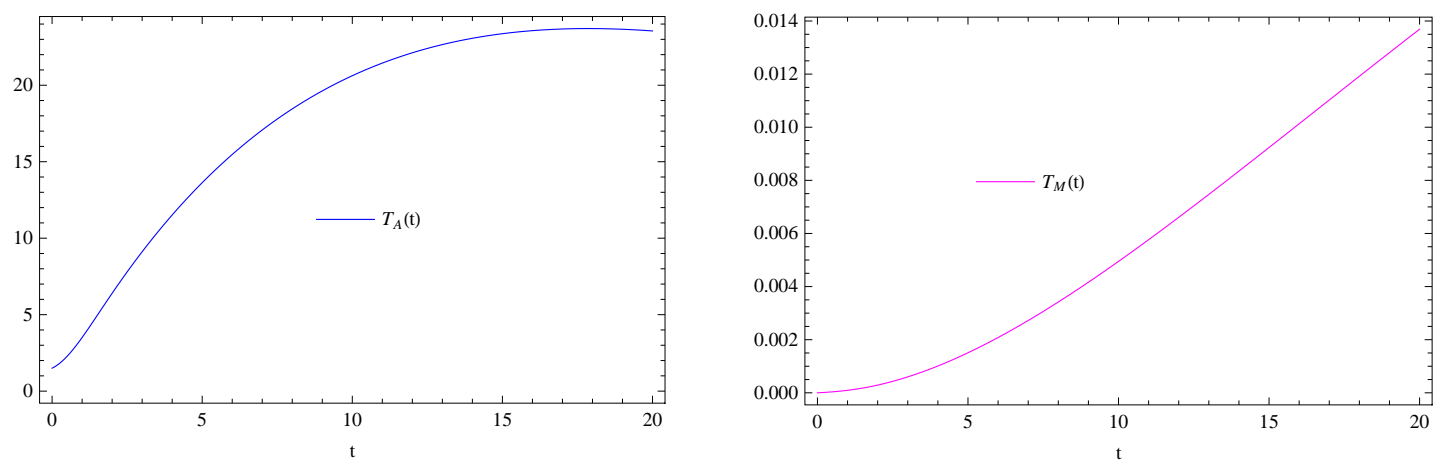

Figure 3. NMG4 solutions of $T_{A}(t)$ for the time in- Figure 4. NMG4 solutions of $T_{M}(t)$ for the time interval $(0,20)$ with the step size $\frac{1}{10}$ for the equation terval $(0,20)$ with the step size $\frac{1}{10}$ for the equation system (14-17) system (14-17) 

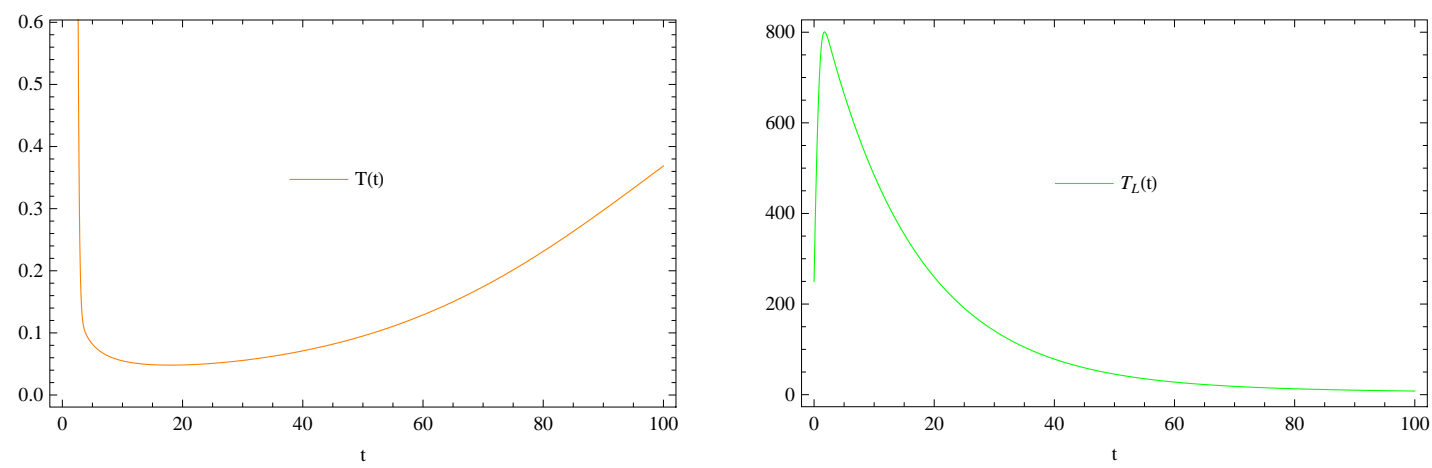

Figure 5. NMG4 solutions of $T(t)$ for the time in- Figure 6. NMG4 solutions of $T_{L}(t)$ for the time interval $(0,100)$ with the step size $\frac{1}{10}$ for the equation terval $(0,100)$ with the step size $\frac{1}{10}$ for the equation system (14-17) system (14-17)
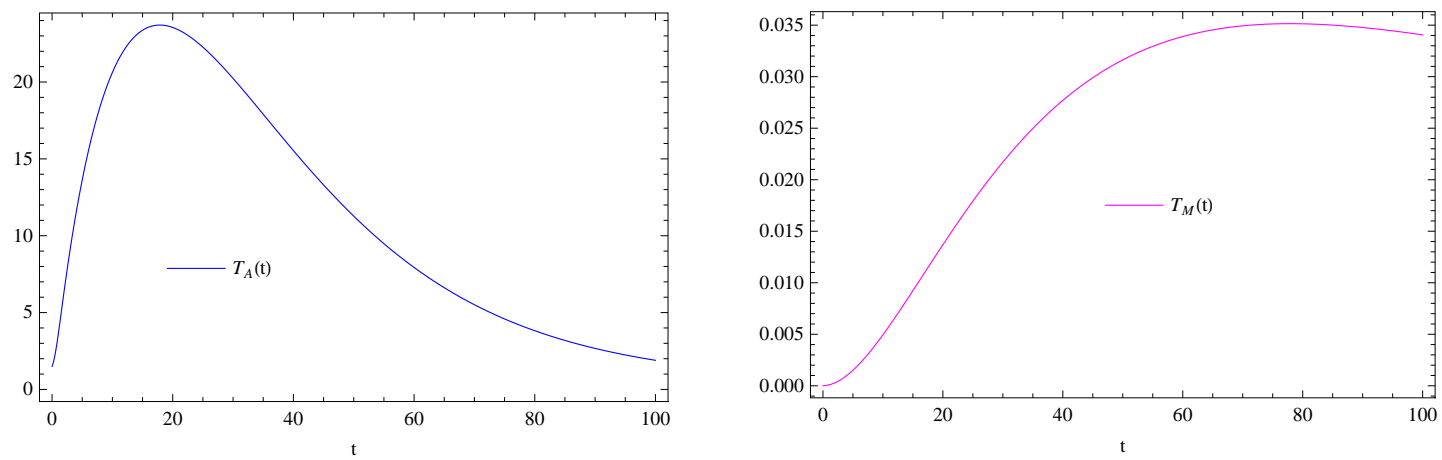

Figure 7. NMG4 solutions of $T_{A}(t)$ for the time in- Figure 8. NMG4 solutions of $T_{M}(t)$ for the time interval $(0,100)$ with the step size $\frac{1}{10}$ for the equation terval $(0,100)$ with the step size $\frac{1}{10}$ for the equation system (14-17) system (14-17)
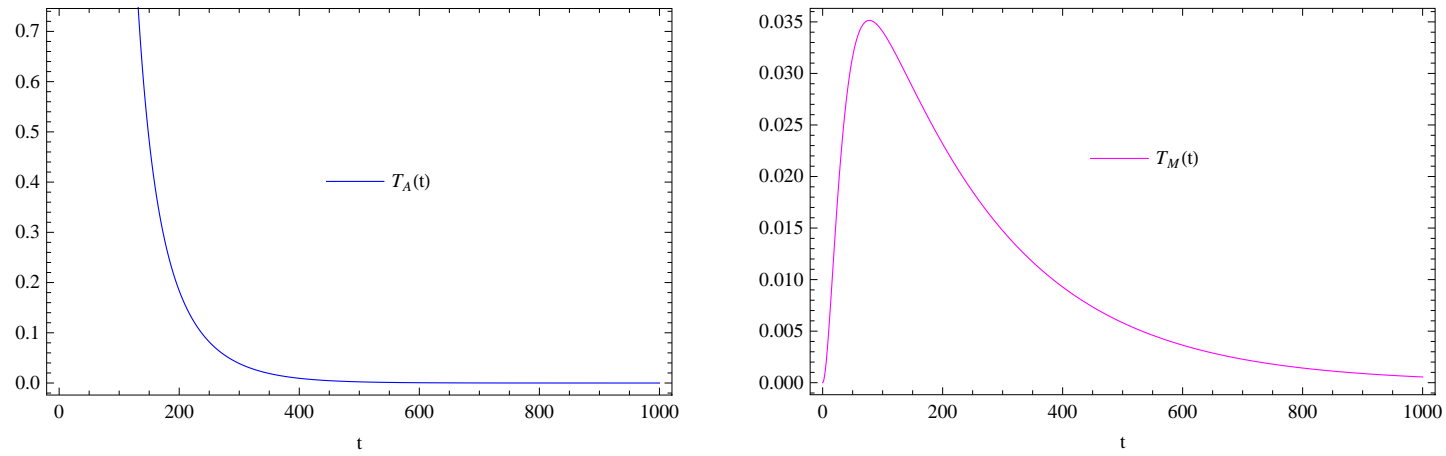

Figure 9. NMG4 solutions of $T_{A}(t)$ for the time in- Figure 10. NMG4 solutions of $T_{M}(t)$ for the time terval $(0,1000)$ with the step size $\frac{1}{10}$ for the equation interval $(0,1000)$ with the step size $\frac{1}{10}$ for the equation system (14-17) system (14-17) 

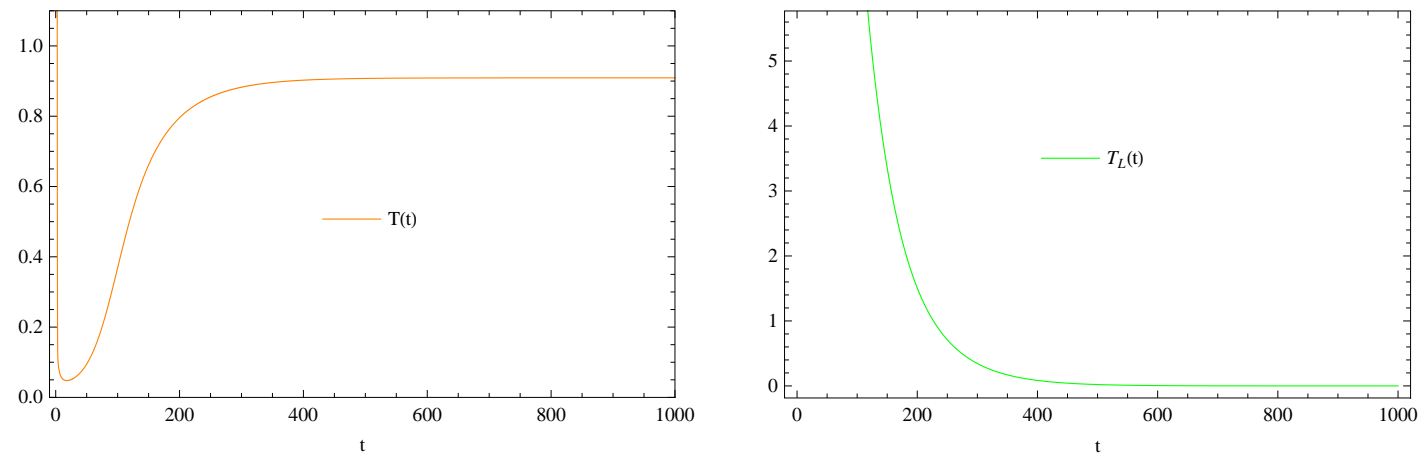

Figure 11. NMG4 solutions of $T(t)$ for the time in- Figure 12. NMG4 solutions of $T_{L}(t)$ for the time terval $(0,1000)$ with the step size $\frac{1}{10}$ for the equation interval $(0,1000)$ with the step size $\frac{1}{10}$ for the equation system (14-17) system (14-17)

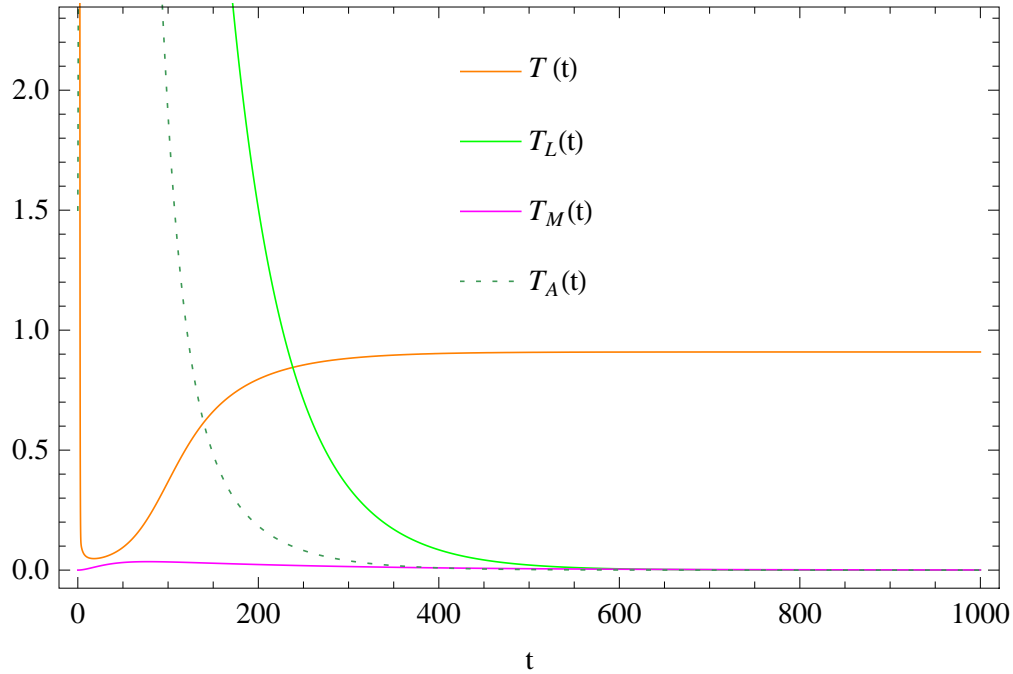

Figure 13. NMG4 solutions of $T(t), T_{L}(t), T_{A}(t)$ and $T_{M}(t)$ for the time interval $(0,1000)$ with the step size $\frac{1}{10}$ for the equation system (14-17)

\section{Conclusion}

In this study, a reliable, efficient and structure preserving [17] numerical algorithm based on the Magnus series expansion is applied to solve the nonlinear differential equation system (14-17) which occurs in Human T-Cell Lymphotropic Virus I (HTLV-I) infection of CD4 ${ }^{+}$T-cells model. The obtained results by NMG4 and the fourth order explicit RungeâAŞKutta (RK45) are compared in Table 1-4. The obtained results are shown in Fig. 1-13. The numerical solutions of the NMG4 are in excellent agreement with respect to the RK45 solutions. As a result Magnus Expansion Method is an efficient and accurate tool for Human T-Cell Lymphotropic Virus I (HTLV-I) infection of CD4 ${ }^{+}$T-cells model. 


\section{References}

1. Gómez-Acevedo, H., Li, M.Y., Backward bifurcation in a model for HTLV-I infection of CD4 ${ }^{+}$T cells, Bulletin of Mathematical Biology, Volume 67: 101-114, (2005).

2. Song, X., Li, Y., Global stability and periodic solution of a model for HTLV-I infection and ATL progression, Applied Mathematics and Computation, Volume 180: 401-410, (2006).

3. Stilianakis, N., Seydel, J., Modeling the T-cell Dynamics and Pathogenesis of HTLV-I Infection, Bulletin of Mathematical Biology, Volume 61: 935-947, (1999).

4. Eshima, N., Tabata, M., Okada, T., Karukaya, S., Population dynamics of HTLV-I infection: a discrete-time mathematical epidemic model approach, Mathematical Medicine and Biology 20(1):29-45, (2003).

5. Seydel, J., Stilianakis, N., HTLV-I Dynamics: A Mathematical Model, Sexually Transmitted Diseases 27(10): 652âĂŞ653, (2000).

6. Wang, L., Li, M.Y. and Kirschner, D., Mathematical analysis of the global dynamics of a model for HTLV-I infection and ATL progression, Mathematical Biosciences 179: 207-217, (2002).

7. Gokdogan, A. and Merdan, M., A multistage homotopy perturbation method for solving Human T-Cell Lymphotropic Virus I (HTLV-I) infection of T-cells model, Middle-East Journal of Scientific Research, 9(4): 503-509, (2011).

8. Magnus, W., On the exponential solution of differential equations for a linear operator, Comm. Pure and Appl. Math. 7: 639-673, (1954).

9. Blanes S. et al., The Magnus expansion and some of its applications, Physics Reports 470: 151-238, (2009).

10. Iserles, A. and Norsett, S.P., On the solution of linear differential equations in Lie groups, Technical Report 1997/NA3, Department of Applied Mathematics and Theoretical Physics, University of Cambridge, England, (1997).

11. Iserles, A. and Norsett, S.P., Linear ODEs in Lie groups, DAMTP tech. report 1997/NA9, University of Cambridge, (1997).

12. Blanes, S. et al., Magnus and Fer expansions for matrix differential equations: the convergence problem, J. Phys. A 31: 259-268, (1998).

13. Moan, P.C. and Niesen, J., Convergence of the Magnus Series, Foundations of Computational Mathematics 8(3): 291-301, (2008).

14. Iserles, A. et al., Lie-group Methods, Acta Numerica 9: 215-365, (2000).

15. Casas, F. and Iserles, A., Explicit Magnus expansions for nonlinear equations, Journal of Physics A: Mathemat Äścal and General 39: 5445â̆̆Ş5461, (2000).

16. Blanes, S. et al., Time-averaging and exponential integrators for non-homogeneous linear IVPs and BVPs, Applied Numerical Mathematics 62: 875-894, (2014).

17. Castellano, A. et al., Geometric numerical integrators based on the Magnus expansion in bifurcation problems for non-linear elastic solids, Frattura ed Integrità Strutturale 29: 128-138, (2014). 